\title{
Molecular dynamics study of isobaric and isochoric glass transitions in a model amorphous polymer
}

\author{
Liu Yang, ${ }^{\text {a) }}$ David J. Srolovitz, and Albert F. Yee \\ Department of Materials Science and Engineering, University of Michigan, \\ Ann Arbor, Michigan 48109-2136
}

(Received 23 December 1997; accepted 29 October 1998)

\begin{abstract}
We perform molecular dynamics simulations of the glass transition through isobaric and isochoric cooling of a model polymeric material. In general, excellent agreement between the simulation results and the existing experimental trends is observed. The glass transition temperature $\left(T_{g}\right)$ is found to be a function of pressure under isobaric conditions and specific volume under isochoric conditions. Under both isobaric and isochoric conditions, the trans-state fraction and the torsional contributions to the energy undergo abrupt changes at the glass transition temperature. We analyze these data to show that the glass transition is primarily associated with the freezing of the torsional degrees of the polymer chains which is strongly coupled to the degree of freedom associated with the nonbonded Lennard-Jones potential. We attribute the greater strength of the glass transition under constant pressure conditions to the fact that the nonbonded Lennard-Jones potential is sensitive to the specific volume, which does not change during cooling under isochoric conditions. Comparison of the isochoric and isobaric data demonstrate that the thermodynamic state is independent of cooling path above $T_{g}$, while path-dependent below $T_{g}$. The simulation data show that the free volume at the isobaric glass transition temperature is pressure dependent. We also find that a glass transition occurs under isochoric conditions, even though the free volume actually increases with decreasing temperature. (C) 1999 American Institute of Physics.
\end{abstract}

[S0021-9606(99)51405-0]

\section{INTRODUCTION}

Glass transition phenomena in amorphous polymers have been of long standing interest to polymer scientists. ${ }^{1-4}$ Besides practical engineering reasons, understanding the glass transition phenomena is central to any explanation of the nature of the glassy state of polymers. In this study, we examine glass transition phenomena of amorphous polymers through molecular dynamics (MD) simulations. Two sets of simulations are performed: Isobaric (constant pressure) and Isochoric (constant volume) cooling with constant cooling rates.

Traditionally, the glass transition of polymers has been experimentally studied through isobaric conditions, during which the volume varies in accordance with the temperature change. Consequently, volume change has often been regarded as an essential factor in explaining the glass formation process. ${ }^{1,2}$ Fox and Flory ${ }^{5}$ first proposed the free volume theory to explain the glass transition. This concept of the glass transition as an iso-free volume state was further applied by Simha and Boyer. ${ }^{6}$ It was assumed that volume inside polymers can be divided as occupied and free volume. When the temperature is decreased to the glass transition temperature, the free volume reaches a critical value, which is insufficient for molecules to adjust, and glass transition occurs. Below the glass transition temperature, both the quantity and the spatial arrangement of free volume remains

${ }^{\text {a) }}$ Current address: NASA/Ames Research Center, Mail Stop 230-3, Moffett Field, CA 94035. Electronic mail: liuyang@pegasus.arc.nasa.gov fixed. Another free volume approach was formed on the basis of the empirical WLF equation, ${ }^{7}$ which was found to describe the viscoelasticity change for the temperature interval $T_{g}$ to $T_{g}+100 \mathrm{~K}$ very well. Doolittle ${ }^{8}$ and $\mathrm{WLF}^{7}$ explained this empirical equation in terms of the decrease of free volume, and predicted a rapid increase of viscosity with decreasing free volume as $T_{g}$ is approached. There are other modifications of the basic free volume theory described above, consequently there is no universally accepted concept of free volume. There exists experimental data showing that system properties can be correlated with changes in the volume. However, the question remains whether the volume change is the only cause for the glass transition or the result of changes in molecular mobility. It is also possible that the system volume and molecular mobility influence each other during the glass transition.

Gibbs and DiMarzio 9 explained the glass transition from a thermodynamics point of view. They employed a statistical-mechanical quasi-lattice theory to calculate the configurational partition function which accounts for the chain stiffness and the volume change with temperature. At a given pressure, the number of allowed arrangements for the molecules decreases with decreasing temperature. This occurs because of two reasons: The decreased volume permits fewer chain configurations; and the chains favor low energy states at lower temperatures. When the configurational entropy becomes zero at a temperature $T_{2}$, a second-order transition occurs. As the temperature approaches $T_{2}$, the system mobility dramatically decreases because of the decreased 
configurational entropy. The observed glass transition temperature $T_{g}$ is considered to be the kinetic reflection of $T_{2}$, which is the underlying thermodynamic transition. Since the predicted $T_{2}$ can only be realized by an infinitely slow cooling rate, its existence cannot be directly examined by experiments or even by computer simulation. However, it will be meaningful to examine the conformational structural changes during the glass transition, so that we may determine if the glass transition is directly related to the freezing of the conformation entropy.

Despite extensive experimental efforts, the glass transition phenomena of polymers are still not fully understood. Important questions remain outstanding because of the inability to access the molecular level information needed to characterize the molecular motion and structural changes during the glass transition and to relate those changes to the underlying (inter- and intramolecular) bonding thermodynamics. Despite the associated length and time scale limitations, simulations can provide detailed structural information on the molecular scale during the glass transition. ${ }^{3}$ Roe and co-workers ${ }^{10,11}$ have performed extensive molecular dynamics studies of the glass transition of small alkane molecules and polyethylene systems. The results demonstrated the feasibility of employing MD to capture glass transition phenomena on the nano-second time scale. They found that the internal energy exhibits a fairly abrupt change at $T_{g}$. These simulations also provided important insight into the molecular scale during the glass transition, such that the conformation transition rate and the segmental diffusion coefficient vanish below $T_{g}$. Brown and Clarke ${ }^{12}$ also found that the torsional component of the conformational state is effectively frozen below $T_{g}$. These results suggested that the glass transition phenomena are closely associated with the freezing of the torsional degrees of freedom. Boyd and coworkers ${ }^{13-15}$ systematically studied the glass transition in several materials. Using calibrated potentials, they demonstrated that despite the upward temperature displacement due to the very short MD time scale, it is possible to make useful material predictions using simulations.

Because of the experimental challenges associated with fixed volume experiments, true isochoric glass transition experiments in amorphous polymers have rarely been performed. Recently, Colucci et al. performed systematic isobaric and isochoric glass formation experiments on polycarbonate. ${ }^{16}$ The strength of the isochoric glass transition (i.e., defined as the ratio between the slopes of the pressure versus temperature curve above and below $T_{g}$ ) is much weaker than the strength of the isobaric glass transition (i.e., defined as the ratio between the slopes of the specific volume versus temperature above and below $T_{g}$ ). No simulations have been performed to date which can be used to understand the differences between the glass transitions under truly isobaric and isochoric conditions. Furthermore, the effect of external pressure under isobaric conditions on glass transition of amorphous polymers have not been explored in previous simulation studies. Motivated by the experimental work of Colucci et al., ${ }^{16}$ we have performed a series of MD simulations cooling the samples through $T_{g}$ under isobaric conditions at different pressures and isochoric conditions at different specific volumes. The purpose of these simulations is several fold. First, under isobaric conditions, we investigate whether the MD simulations can reproduce the experimental trends in the glass transition behavior with pressure despite the fact that the time scales for the MD simulations (nano-seconds) are very much shorter than the experimental ones. Second, we investigate whether the MD simulations can capture the much weaker glass transition signatures seen in the recent isochoric glass transition experiments. ${ }^{9}$ Above all, the focus of this work is to understand the thermodynamics and kinetics of the glass transition by comparing glass formation under isobaric and isochoric conditions. To aid in this effort, we examine the roles played by the different types of atomic interactions that are important in polymeric systems.

We employed a set of potentials that closely resemble the structure and properties of polyethylene (PE). The interactions in model system, the simulation method, and the sample preparation procedure will be described in the next section. The isobaric and isochoric results are presented in Secs. III and IV, respectively. In Sec. V, we examine and compare the results of the two types of simulations to better understand the glass transition and the free volume theory often used to describe it. Finally, we conclude our study in Sec. VI.

\section{SIMULATION DETAILS AND SAMPLE PREPARATION}

Molecular dynamics (MD) simulations yield dynamical information about the system by explicitly integrating the equations of motion for all the particles in the system. The behavior of the polymeric system within the framework of the MD simulations is determined by three main factors: (1) The atomic interactions, (2) the equations of motion and the constraints on these motions employed to fix the thermodynamic ensemble (and the numerical methods to integrate them), and (3) the initial molecular structure of the system.

\section{A. Interactions}

The purpose of the present study is to investigate the glass transition and the effect of thermodynamic ensemble on it, rather than to examine the glass transition behavior of a particular polymer. As long as the interatomic potentials capture the main features of the interactions in polymers, they should serve this purpose. We employ a set of interatomic potentials fit to $\mathrm{PE}$. The amorphous $\mathrm{PE}$ is described in terms of the united atom model, in which each $\mathrm{CH}_{2}$ group is considered as one united atom. Since the intrachain covalent bond potential is, by far, the stiffest interaction in the polymer system, it largely limits the time step employed in an MD simulation. The amplitude of the vibration along the bond direction is very small due to this stiffness and contributes little to chain configuration changes. Therefore, the intrachain bonds are constrained to a fixed length of $0.153 \mathrm{~nm}$. The remaining interactions are described by the nonbonded Lennard-Jones (LJ) potential, and bond bending (three-body) and torsional (four-body) potentials. We employ the same forms of these potentials and parameters as did Brown and Clarke, ${ }^{12}$ with the exception that we modified the LJ poten- 
tial to insure a smooth cutoff at $2.5 r_{0}$ for the potential and its first derivative, where $r_{0}$ is the Lennard-Jones length parameter. The modified LJ potential acts on all pairs of united atoms, except those intrachain neighboring pairs separated by less than four bonds. The well-depth of the LJ potential is $k_{B} T_{0}$, where $T_{0}=57 \mathrm{~K}$ and $k_{B}$ is Boltzmann's constant. The $\mathrm{LJ}$ length parameter is $0.428 \mathrm{~nm}$. The bending potential is written in terms of the valence angle $\theta: \Phi_{\text {bending }}(\theta)$ $=\frac{1}{2} k_{\theta}\left(\cos \theta-\cos \theta_{0}\right)^{2}$, where $k_{\theta}=520 \quad \mathrm{~kJ} / \mathrm{mole}$ and $\theta_{0}=112.813^{\circ}$. The torsional potential is a four-body potential written in terms of the dihedral angle $\phi: \Phi_{\text {torsion }}(\phi)$ $=C_{0}+C_{1} \cos \phi+C_{2} \cos ^{2} \phi+C_{3} \cos ^{3} \phi$, where $C_{i}, i=0,3$ are $8.832,18.087,4.880$, and $-31.800 \mathrm{~kJ} / \mathrm{mole}$, respectively. Note that the bending potential is much stiffer than the torsional one. The trans state of the dihedral angle, where $\phi=0^{\circ}$, gives the lowest torsional potential energy; and the gauche-state, where $|\phi| \approx 112^{\circ}$, is a metastable state with a potential energy of $4.42 \mathrm{~kJ} / \mathrm{mole}$; there is a potential barrier of $15.12 \mathrm{~kJ} / \mathrm{mole}$ at $|\phi| \approx 60^{\circ}$. These potential features play important roles in the glass transition, discussed below. In the analysis presented here, we consider a bond triplet to be in the trans orientation when the absolute value of $\phi$ is less than $60^{\circ}$.

\section{B. Simulation details}

Since the glass transition is a kinetic process, the path the system traverses is important in determining the structure of the glass and the nature of the glass transition. We employ Andersen and Nosé's extended ensemble methods to control the system pressure and temperature ${ }^{17,18}$ and SHAKE $^{19-21}$ algorithm to constrain the bond lengths in our NPT and NVT molecular dynamics simulations. The NPT MD equations of motion for the molecular system with bond length constraints were derived by Ferrario and Ryckaert. ${ }^{21}$ This set of equations dynamically control the system temperature and pressure at the specified value $T_{\text {ext }}$ and $P_{\text {ext }}$. Therefore, the system is under isobaric conditions with a specified temperature. To simulate the isochoric conditions, volume change is set to zero. These equations reduce the NPT ensemble to the NVT ensemble. By decreasing the target temperature $T_{\text {ext }}$ with a fixed rate, isobaric or isochoric cooling at fixed $d T_{\text {ext }} / d t$ is achieved, under the assumption that the simulation system can rapidly adjust to the changes in $T_{\mathrm{ext}}$. For the value of $d T_{\text {ext }} / d t$ employed in the present simulations $\left(10^{-3}\right.$ degrees per MD time step), the system temperature and $T_{\text {ext }}$ accurately and promptly track each other. With this cooling rate the temperature can be reduced from 500 to $100 \mathrm{~K}$ in $4 \times 10^{5} \mathrm{MD}$ time steps ( $\approx 1$ nanosecond). Performing similar simulations at higher cooling rates yielded no qualitative changes in the glass transition behavior but did lead to a slightly less dense glass. The present glass transition simulation in polymers differs from previous studies ${ }^{10-15}$ in that it is performed at constant cooling rate under rigorously isobaric (for several pressures) or isochoric (at two volumes) conditions. The numerical algorithm employed in our study to integrate the equations of motion is described in details in Ref. 22. The inertia parameters associated with volume and temperature fluctuations are set to $w s=2.0 \times 10^{3}$ a.m.u. $\AA$ and $w Q=1.5$ a.m.u. $\AA^{-3}$, respectively. While there is no rigorous physical basis to guide the choice of these parameters, we chose these values to insure that the system equilibrates quickly without unphysically large fluctuations. We employ a time step of $d t=\tau=2.63 \times 10^{-15}$ s (i.e., $2.63 \mathrm{fs}$ ) in the present simulation. Parallel computation was used. The simulation program was fully parallelized using two types of decomposition (atomic and molecular decomposition) and two message passing events (force calculation and position update) between the nodes in each MD step. Atomic decomposition was employed in both the neighbor list construction for the nonbonded $\mathrm{LJ}$ force calculation and in the $\mathrm{LJ}$ force calculation. For bonded force and bond length constraint force calculations, molecular decomposition was employed.

Accurate determination of the pressure is critical for both the isobaric (as a control parameter) and isochoric (as a measurement) glass formation studies. In a molecular system, the pressure can be calculated based upon either molecular or atomic units. The average value of the pressure calculated using these two approaches should be equal. This equivalence has been demonstrated by Berendsen. ${ }^{23}$ Nonetheless, the instantaneous values of the pressure calculated using these two approaches are not necessarily the same. In our simulation, the pressure has been calculated using the molecular approach by summing the following expression over all $N$ molecules: $P=\sum_{\alpha=1}^{N}\left(\mathbf{P}_{\alpha}^{2} / M+\mathbf{F}_{\alpha} \mathbf{R}_{\alpha}\right)(3 V)^{-1}$, where $\mathbf{P}_{\alpha}, \mathbf{F}_{\alpha}$, and $\mathbf{R}_{\alpha}$ are the momenta, forces and coordinates of the molecular centers of mass and $V$ is the system volume. Periodic boundary conditions are employed in all three orthogonal directions in the present simulations. For the data reported here, the simulation cell includes five polymer chains where each chain contains 300 united atoms, each of which represents one $\mathrm{CH}_{2}$ unit. Roe and co-workers ${ }^{10}$ showed that for chains longer than $\sim 200$ units, the glass transition behavior is relatively insensitive to further increases in the chain length. The chains are not confined to the simulation cell but, instead, traverse several periodic cell images. The simulation cell volume was $\sim 50 \mathrm{~nm}^{3}$ at $100 \mathrm{~K}$. In order to improve the statistics, calculation over three independent samples were performed and averaged for the isobaric study and six for the isochoric study.

\section{Sample preparation}

The thermal history of an amorphous polymer is important in determining its structure and properties. A series of high temperature, liquid samples must first be prepared before the cooling simulations may be performed. The mobility of the polymer chains in the melt is small compared to that in a small molecule system. Even at high temperatures, an individual chain can only explore a small portion of the conformational phase space during the course of a typical MD simulation. Therefore, our initial guess as to the structure of the polymer melt is important for the final melt (and glass) structure. We obtain the initial structure using the phantom chain growth with excluded volume method introduced by McKechnie et $a l .{ }^{24}$ During chain growth, the bending angles are fixed at their equilibrium value and the torsional angles 
are selected based upon a Monte Carlo procedure which considers only the torsional potential and the fourth intrachain neighbor LJ potential. This Monte Carlo procedure prevents the growth of high energy chain conformations. The phantom chain growth procedure produces an initial structure with a density of $\sim 0.7 \mathrm{~g} / \mathrm{cm}^{3}$ at $500 \mathrm{~K}$, which is well above the $T_{g}$. The nonbonded LJ interactions not taken into account during the chain growth can lead to very high energies and large forces which could cause numerical instabilities in the MD program. To avoid this, a softened LJ potential, which gives a constant force for the atomic separation below $0.85 r_{0}$, is employed to slowly introduce the appropriate excluded volume into the system during the first 500 MD steps. The simulation cell volume is kept constant during this relaxation phase. The extremely large internal pressure associated with the initial guess structure is effectively reduced during this $500 \mathrm{MD}$ step relaxation procedure.

Following the initial relaxation, the full interactions are switched on. The isobaric samples are further relaxed by holding at a pressure of one bar and a temperature of $500 \mathrm{~K}$ for $\sim 0.8$ nanoseconds with the NPT control. The system volume quickly equilibrates and then fluctuates around the equilibrium value. Since one bar $(\approx 0.1 \mathrm{MPa})$ is very close to zero compared to the other pressure conditions employed for the isobaric study (100 and $200 \mathrm{MPa})$, these samples are effectively at zero pressure. Samples under other isobaric conditions $(P=100$ or $200 \mathrm{MPa})$ are prepared by gradually increasing the pressure of the zero pressure samples at a constant rate, while keeping the temperature fixed at $500 \mathrm{~K}$. This is accomplished within the NPT extended ensemble MD method by increasing the target pressure $P_{\text {ext }}$ at a rate of $d P_{\text {ext }} / d t=5 \times 10^{-4} \mathrm{MPa} / \tau$. After reaching the target pressure, the samples are allowed to further relax at $500 \mathrm{~K}$ at this pressure for $10^{5} \mathrm{MD} \tau$ (0.26 nanoseconds). Since the pressure is increased slowly during this procedure, the system effectively tracks the target pressure $P_{\text {ext }}$. Holding the sample at the final pressure and temperature has no discernible effect on sample properties, thereby indicating the effectiveness of this equilibration procedure. Specific volumes of 1.250 and $1.275 \mathrm{~cm}^{3} / \mathrm{g}$ are chosen for the isochoric study. The rationale behind these choices will become evident below. Since the $200 \mathrm{MPa}$ isobaric samples have specific volumes closest to these two target volumes, the isochoric samples were prepared from the $200 \mathrm{MPa}$ isobaric samples by compressing or expanding to the system volume at a rate of $|\Delta V| / V=10^{-6} \tau^{-1}$. After achieving the desired specific volume, the system is held for $5 \times 10^{5} \tau$ (0.8 nanoseconds) to further equilibrate.

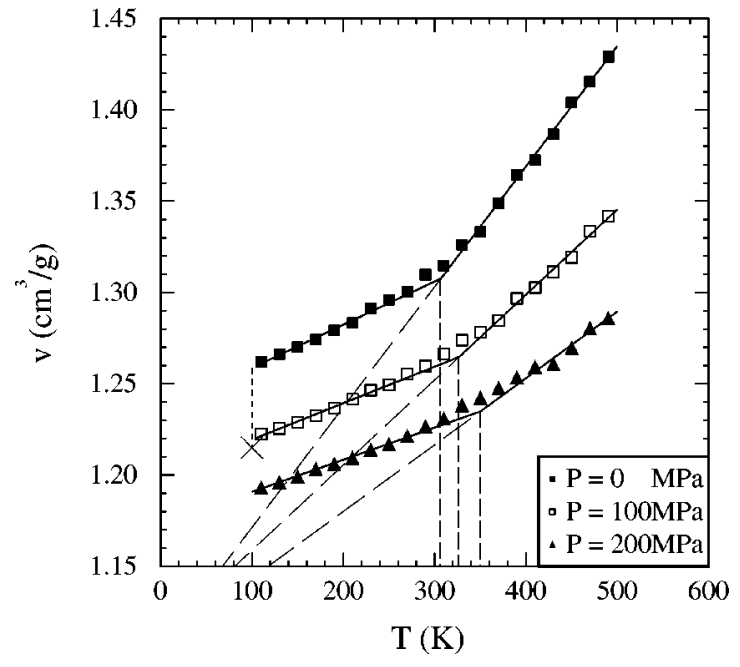

FIG. 1. The specific volume vs temperature for isobaric cooling at three different pressures. The symbols represent the simulation data, the solid lines represent the best linear fits (see Table I) and the long dashed lines are extrapolations of the high temperature solid lines to below the glass transition. The short dashed lines indicate $T_{g}$ 's for each pressure. The vertical dotted line and the " $X$ " indicate the specific volume found when the zero pressure system is compressed to $P=200 \mathrm{MPa}$ at $100 \mathrm{~K}$.

\section{ISOBARIC GLASS FORMATION}

The specific volume, $\nu$, versus temperature, $T$, for isobars at pressures of zero, 100 and $200 \mathrm{MPa}$ are presented in Fig. 1. The symbols in the plot represent the simulation data averaged over a 20 degree temperature interval. Unless specified, this same averaging procedure is used throughout. At both high and low temperatures, the specific volume decreases linearly with decreasing temperature for all three pressures, albeit the thermal expansion coefficients are smaller at low temperature. The abrupt change in the thermal expansion coefficients in Fig. 1 between 300 and $350 \mathrm{~K}$ is a classical signature of the glass transition. These data suggest that $T_{g}$ of this model system increases from $\sim 300$ to $350 \mathrm{~K}$ as the pressure is increased from 0 to $200 \mathrm{MPa}$. To determine the glass transition temperature $T_{g}$ and glass formation volume $v_{g}$, a least square linear fit to the form $v=a T+b$ is made for the data at temperatures well above and below $T_{g}$. The intersection of the high and low temperature linear fits (for each pressure) is taken as $T_{g}$. The temperature range for the linear fitting of each of the lines and the fitting coefficients are presented in Table I, in which the subscript " $r$ ", indicates the parameters for the rubbery state, and the subscript " $g$ " for the glassy state. The specific volume at zero

TABLE I. Parameters obtained from fitting the specific volume $v$ vs $T$ (see Fig. 2) for the isobaric cooling data.

\begin{tabular}{|c|c|c|c|c|c|c|c|c|c|}
\hline \multirow[b]{2}{*}{$P$} & \multicolumn{3}{|c|}{$T<T_{g}$} & \multicolumn{3}{|c|}{$T_{g}$} & \multicolumn{3}{|c|}{$T>T_{g}$} \\
\hline & $\begin{array}{c}\text { Fitting } \\
\text { range }(\mathrm{K})\end{array}$ & $a_{g} \times 10^{4}$ & $b_{g}$ & $T_{g}(\mathrm{~K})$ & $v_{g}$ & $\begin{array}{c}\text { Strength } \\
a_{g} / a_{r}\end{array}$ & $\begin{array}{c}\text { Fitting } \\
\text { range }(\mathrm{K})\end{array}$ & $a_{r} \times 10^{4}$ & $b_{r}$ \\
\hline $0 \mathrm{Mpa}$ & $100-240$ & $2.37 \pm 0.12$ & $1.235 \pm 0.002$ & 306 & 1.307 & 2.78 & $320-500$ & $6.59 \pm 0.16$ & $1.105 \pm 0.006$ \\
\hline $100 \mathrm{MPa}$ & $100-260$ & $1.99 \pm 0.06$ & $1.200 \pm 0.001$ & 326 & 1.265 & 2.35 & $360-500$ & $4.67 \pm 0.21$ & $1.112 \pm 0.009$ \\
\hline $200 \mathrm{MPa}$ & $100-280$ & $1.76 \pm 0.03$ & $1.173 \pm 0.001$ & 350 & 1.235 & 2.07 & $400-500$ & $3.65 \pm 0.43$ & $1.107 \pm 0.020$ \\
\hline
\end{tabular}


pressure in Fig. 1 is larger (by $\sim 0.1 \mathrm{~cm}^{3} / \mathrm{g}$ ) than the experimental values of amorphous PE, extrapolated from densities of the semicrystalline PE. This discrepancy may be due to the fast quenching rate employed in the MD simulation. Another and more likely reason can be attributed to the interatomic potentials used here do not accurately represent PE.

Several important observations can be made on Fig. 1. First, the glass transition temperature $T_{g}$ increases and the glass formation volume $v_{g}$ decreases with increasing pressures. Second, the slope of the specific volume versus temperature in the rubbery state, $a_{r}$, decreases with increasing pressure. The linear curve fits from the rubbery state at different pressures all extrapolate to the same specific volume at $0 \mathrm{~K}, v_{0}=1.11 \mathrm{~cm}^{3} / \mathrm{g}$, within the precision of the data (see $b_{r}$ in Table I). All these are consistent with experimental observations of several systems (e.g., polystyrene ${ }^{25}$ and polycarbonate $\left.{ }^{16}\right)$. Third, the slope of specific volume versus temperature in the glassy range, $a_{g}$, decreases with increasing pressure, although this is much weaker than in the rubbery state. Unlike for the rubbery state, the glassy state data do not extrapolate to the same specific volume at $0 \mathrm{~K}$. As suggested by Colucci et al., ${ }^{16}$ the ratio of the slopes of the specific volume versus temperature lines for the rubbery and glassy states, $a_{\mathrm{r}} / a_{g}$, provides a measure of the glass transition strength. The data in Table I show that the glass transition strength in the present isobaric simulations decreases with increasing pressure. Finally, we note that the data in Fig. 1 near $T_{g}$ lie above the intersection of the extrapolation of the high and low temperature data. This too is consistent with the experimental observations (see, e.g., Chap. 1, Fig. 3 of Ref. 1). All these observations demonstrate that the MD simulations can capture the experimental trends in glass transition behavior under isobaric conditions.

Above $T_{g}$, the polymer melt is in either equilibrium (above $\left.T_{m}\right)$ or a deep metastable state $\left(T_{g}<T<T_{m}\right)$. When the melt is cooled below $T_{g}$, its mobility rapidly decreases and the departure from equilibrium becomes increasingly severe. This picture is reflected by the observations made with respect to Fig. 1. The observation that the specific volume simulation data lie above the high and low temperature extrapolations near $T_{g}$ is a result of the fact that as the system nears $T_{g}$, the freezing out of configurational degrees of freedom prevents the system from keeping up with the evolving thermodynamic state. The high rate cooling in the MD simulations accentuates this effect. As the pressure is increased, this deviation becomes even more pronounced due to the fact that the relaxation rate slows with increasing density. Another indication of the dependence of the frozen-in structure on the pressure is the variation of the specific volume with pressure. If all of the variations of the specific volume with pressure was associated with the finite compressibility of the glass, then applying a pressure of $200 \mathrm{MPa}$ to the $0 \mathrm{MPa}$ glass would decrease its specific volume to the same value as for the $200 \mathrm{MPa}$ glass. We performed this simulation at 100 $\mathrm{K}$ and the resultant specific volume is indicated by the " $X$ ", in Fig. 1. The resultant specific volumes differ by $\sim 2 \%$. The low density of the low pressure structure is partially preserved upon compression at $100 \mathrm{~K}$, since the structure is essentially frozen well below $T_{g}$.
$\mathrm{T}(\mathrm{K})$

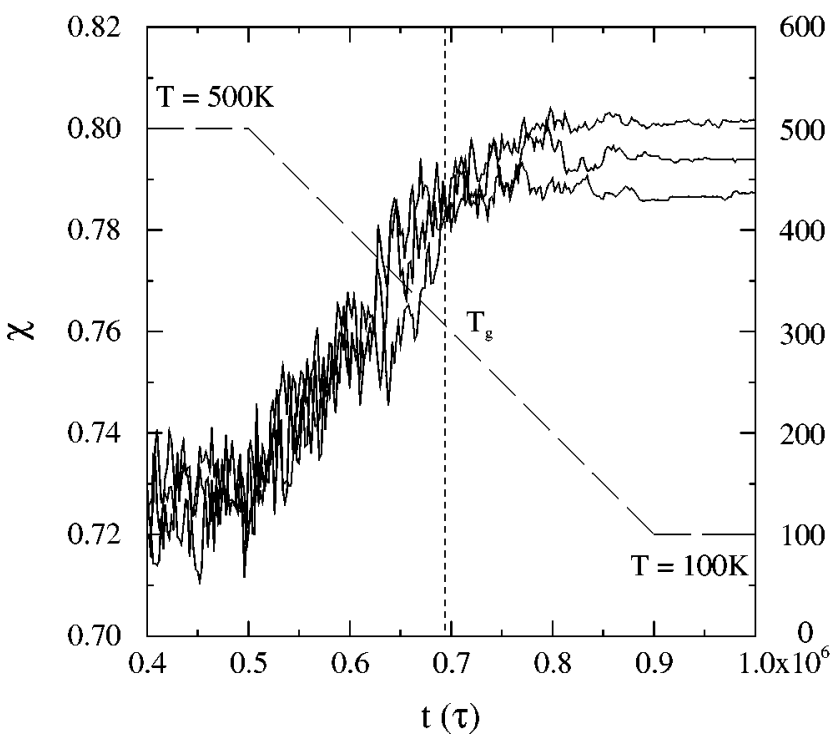

FIG. 2. The trans fraction $\chi$ (solid lines) and temperature (dashed line) vs time for a zero pressure sample held at $500 \mathrm{~K}$, cooled to and held at $100 \mathrm{~K}$. Each data point is averaged over a period of $2 \times 10^{3} \tau$. The three solid lines represent the results of three simulation runs. The dotted line indicates the time at which the temperature crosses $T_{g}$, as per Fig. 1.

One indication of the degree of relaxation in the glass is the fraction of the bonds in the trans state, $\chi$. Figure 2 shows the variation in $\chi$ as the polymer is cooled from $500 \mathrm{~K}$ to below $T_{g}$ and held at $100 \mathrm{~K}$ under a zero pressure. $\chi$ varies little during isothermal holding at $500 \mathrm{~K}$, indicating the equilibrium. During cooling, $\chi$ increases monotonically. In the rubbery state, $\chi$ obtained from three individual simulations are indistinguishable aside from the thermal fluctuations. However, when the system is cooled toward $T_{g}$, the value of $\chi$ asymptotically approaches a temperature independent value. At $T_{g}$, the three simulation curves become distinguishable, indicating that the value of $\chi$ for the glass is determined by the nature of the thermal fluctuations as the system passes through $T_{g} \cdot \chi$ does not vary much with time during further cooling below $T_{g}$ or in holding at $100 \mathrm{~K}$. This demonstrates that conformational fluctuations are effectively frozen out below $T_{g}$. This trend is consistent with the transstate fraction results obtained by previous simulation work of Brown and Clark. ${ }^{12}$ The pressure effects on the variation of $\chi$ with temperature (under isobaric conditions) are shown in Fig. 3. $\chi$ increases rapidly with decreasing temperature and is relatively insensitive to pressure above $T_{g}$. Below $T_{g}, \chi$ asymptotically approaches a constant value. Increasing pressure corresponds to a decreasing asymptotic value of $\chi$. The pressure independence of $\chi$ at high temperatures suggests that pressure has little effect on the equilibrium conformational state. The fact that $\chi$ is sensitive to pressure at lower temperatures demonstrates how pressure makes it more difficult to kinetically explore different conformational states. This can be attributed to the fact that increasing pressure leads to higher densities and stronger nonbonded interactions that resist bond rotation, as discussed below. The pressure dependence of the low temperature asymptote of $\chi$ is a mani- 


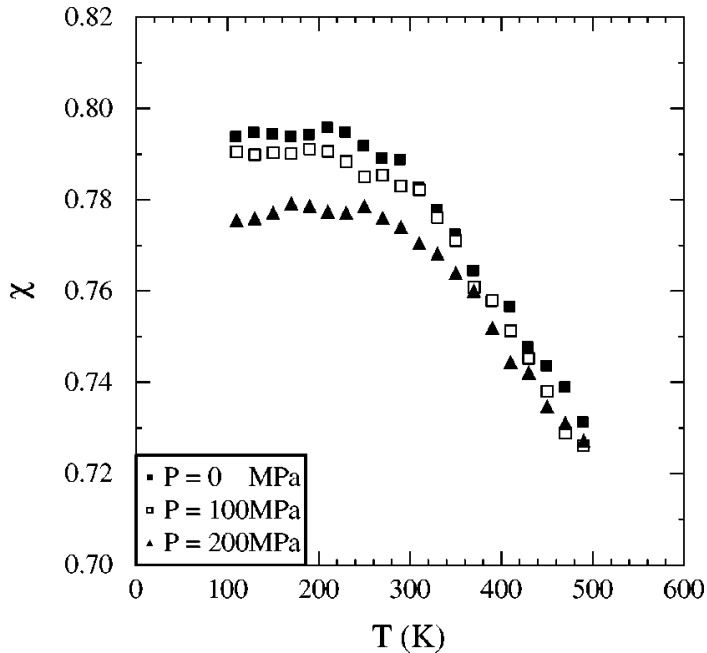

FIG. 3. The trans fraction $\chi$ vs temperature for $P=0,100$, and $200 \mathrm{MPa}$ isobaric simulations.

festation of the fact that the conformational fluctuations are frozen out at higher temperatures with increasing pressure.

To separate the roles played by the different interaction components in the glass transition, the bending, torsional and nonbonded LJ potential energy as a function of temperature during isobaric cooling are plotted in Fig. 4. The bending energy decreases linearly with decreasing temperature and is independent of pressure at all temperatures. It is almost completely insensitive to the glass transition, which suggests that the bending degree of freedom is in equilibrium both in the rubbery and glassy states. This is further supported by the observation that the bending energy extrapolates to near zero at zero temperature. This is also consistent with the experimental observation that the bending spectrum for amorphous polymers changes little between the melt and glassy states. The near equilibration of the bending degrees of freedom at glassy state may be traced to two distinct causes. First, the bending potential is very stiff. Second, only small amplitude atomic motions are required to change the bond angle. In Fig. 4, the torsional energy decreases linearly with decreasing temperature and is independent of pressure above $T_{g}$. It also decreases linearly with decreasing temperature below $T_{g}$ albeit with a smaller slope and a pressure dependent intercept. This is consistent with the temperature dependence of $\chi$ shown in Fig. 3. As discussed with reference to that figure, above $T_{g}$, the thermal fluctuations are sufficient to allow the conformational fluctuations and equilibration of the torsional degrees of freedom; below $T_{g}$, conformational fluctuations do not occur at a sufficient rate to equilibrate these degrees of freedom. This freezing of the conformational degrees of freedom below $T_{g}$ is the origin of glass transition, perhaps in all polymers. While trans-gauche transitions do not occur at an appreciable rate below $T_{g}$, thermal fluctuations still cause small fluctuations in the torsional angles. This accounts for the linear variation of the torsional energy with temperature below $T_{g}$. The variation of the nonbonded LJ potential energy with temperature in Fig. 4 is very similar to that for the specific volume (Fig. 1). Furthermore, like for the specific volume data, the rubbery-state LJ energy data at different

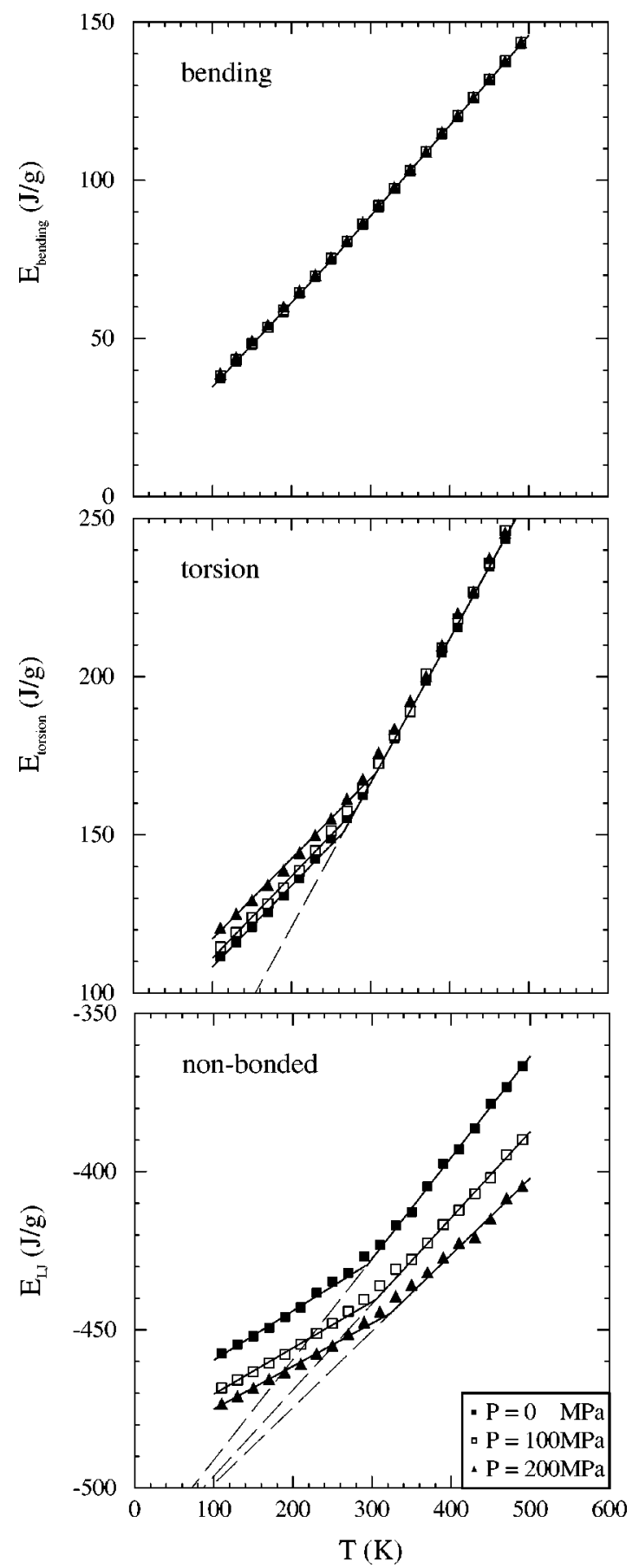

FIG. 4. The bending, torsional and nonbonded LJ energies vs temperature. The symbols represent the simulation data and the solid lines represent least square linear fits to the data in the rubbery and glassy states. The dashed lines are extrapolations of the high temperature fits to below $T_{g}$. The same temperature ranges were used for the fitting here as in Fig. 1 (see Table I).

pressures all extrapolate to the same value at zero temperature. This similarity is not surprising since the nonbonded LJ potential energy directly reflects the average packing in the system. The abrupt change in slope of the LJ energy with temperature at $T_{g}$ is also very similar to the temperature dependence of the torsional energy. This observation suggests that there is a strong coupling between the volume change and freezing of the torsional degrees of freedom at $T_{g}$. As the system contracts during cooling, the chains get 


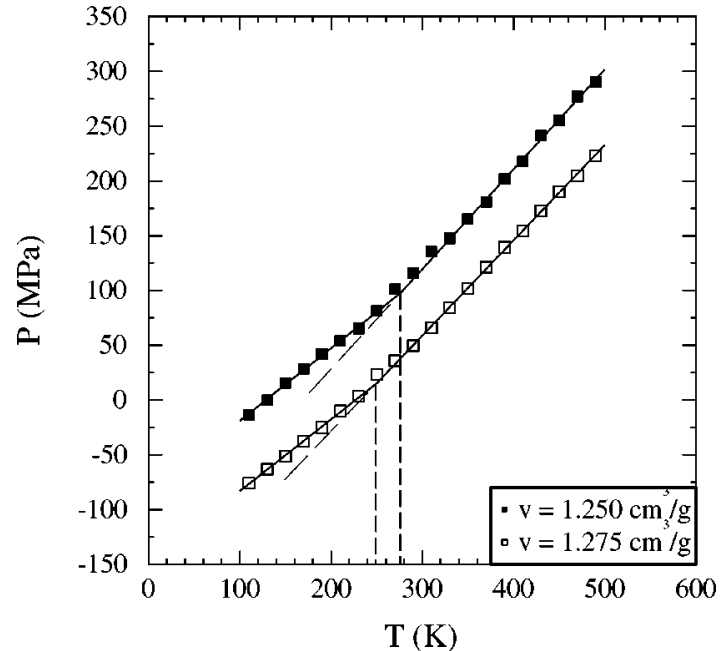

FIG. 5. The pressure vs temperature for isochoric cooling at two specific volumes. The symbols represent the simulation data, the solid lines represent the best linear fits (see Table II) and the long dashed lines are extrapolations of the high temperature solid lines to below $T_{g}$. The short dashed lines indicate $T_{g}$ 's for each specific volume.

closer and closer together until there is no longer sufficient room for torsional state changes to occur. Therefore, the glass transition occurs apparently because of the volume change, which causes the freezing out of the torsional degrees of freedom. We return to this point below in the discussion section. Once the torsional degrees of freedom are frozen out, the chains become more rigid and, hence, more difficult to compress, resulting in a decreased $d V / d T$ below $T_{g}$.

\section{ISOCHORIC GLASS FORMATION}

The dependence of the pressure on temperature for isochoric (fixed volume) cooling is shown in Fig. 5 for specific volumes of 1.250 and $1.275 \mathrm{~cm}^{3} / \mathrm{g}$. These two specific volumes were chosen, based on the isobaric data in Fig. 1, such that the glass transition temperature would be close to the isobaric glass transition temperature and the associated pressures near those examined in the isobaric studies. This allows a ready comparison between the isobaric and isochoric results. The trends in the $P$ vs $T$ data exhibit abrupt changes between 250 and $300 \mathrm{~K}$, indicative of glass transitions. Linear fits of the isochoric data to the form $P=a T+b$ in the rubbery and glassy state are indicated by the solid lines in Fig. 5 and used to determine the glass transition temperature. The parameters $(a$ and $b)$ in the fits, the glass transition temperatures, $T_{g}$, and the glass formation pressure, $P_{g}$, are all listed in Table II. The slopes $(a)$ for both the rubbery and glassy states are relatively insensitive to changes in specific volume. The strengths of the glass transitions, defined as the ratio of the slopes $d P / d T$ for the rubbery and glassy states, $a_{r} / a_{b}$, are also listed in Table II. Comparison of these strengths with those from the isobaric simulations (Table I) indicates that the isochoric glass transitions are much weaker than the isobaric ones. The variation of the strength with specific volume in the isochoric simulations is much weaker than the variation in the strength with pressure in the isobaric simulations, despite the fact that the specific volume and pressure ranges are comparable.

For the same pressure and density conditions, the isochoric glass formation temperatures are lower than in the isobaric simulations. In isobaric cooling (Fig. 1) simulations, the glass formation volume $v_{g}$ is an approximately linear function of the glass formation temperature $T_{g}$. Linear least square fits of the three isobaric $T_{g}$ 's yields $v_{g}$,isobaric $=1.80-0.00162 * T_{g}$, isobaric (in $\mathrm{cm}^{3} / \mathrm{g}$ when $T_{g}$,isobaric is in Kelvin). This can be inverted to yield $T_{g \text {,isobaric }} \approx 339$ and 324 $\mathrm{K}$ at $v_{g \text {,isobaric }}=1.250$ and $1.275 \mathrm{zcm}^{3} / \mathrm{g}$, respectively. However, Fig. 5 (as listed in Table II) shows that the $T_{g}$ 's obtained from the isochoric simulations occur at, $T_{g}$,isochoric $\approx 276$ and $249 \mathrm{~K}$ at $\nu=1.250$ and $1.275 \mathrm{~cm}^{3} / \mathrm{g}$, respectively. The difference between the isobaric and isochoric glass transition temperatures at the same volumes is in excess of $60 \mathrm{~K}$, which is much larger than the uncertainty associated with data fitting. The $T_{g}, v_{g}$, and $P_{g}$ experimental data obtained by Colucci et al. ${ }^{16}$ for polycarbonate shows almost no dependence on the cooling path. Part of this discrepancy may be associated with the much higher cooling rates in the simulations. This is consistent with our observations (and those of many experimental studies) that $T_{g}$, increases with increasing cooling rate for both isobaric and isochoric paths and that the rate of change is not identical for both paths.

Figure 6 shows the variation of the trans-state fraction, $\chi$, with temperature for two specific volumes. As in the isobaric simulations, $\chi$ increases rapidly with decreasing temperature and levels off at a temperature approximately equal to $T_{g}$. In the rubbery state, the values of $\chi$ are nearly indistinguishable for the two values of the specific volume examined here. In the glassy state, $\chi$ is slightly smaller in the low specific volume $\left(1.250 \mathrm{~cm}^{3} / \mathrm{g}\right)$ simulations than in the high specific volume $\left(1.275 \mathrm{~cm}^{3} / \mathrm{g}\right)$ ones. As in the isobaric case, the slowing down of conformation state changes correlate with the onset of $T_{g}$. The slightly lower trans-state fraction $\chi$ at a specific volume of $1.250 \mathrm{~cm}^{3} / \mathrm{g}$ is consistent with the observation that $T_{g}$ is higher at lower specific volume (1.250 $\left.\mathrm{cm}^{3} / \mathrm{g}\right)$ than at higher specific volume $\left(1.275 \mathrm{~cm}^{3} / \mathrm{g}\right)$.

The variation of the bending, torsional and nonbonded

TABLE II. Parameters obtained from fitting the pressure $P$ vs $T$ (see Fig. 6) for the isochoric cooling data.

\begin{tabular}{|c|c|c|c|c|c|c|c|c|c|}
\hline \multirow[b]{2}{*}{$\nu$} & \multicolumn{3}{|c|}{$T<T_{g}$} & \multicolumn{3}{|c|}{$T_{g}$} & \multicolumn{3}{|c|}{$T>T_{g}$} \\
\hline & $\begin{array}{c}\text { Fitting } \\
\text { range }(\mathrm{K})\end{array}$ & $a_{g}$ & $b_{g}$ & $T_{g}(\mathrm{~K})$ & $P_{g}$ & $\begin{array}{c}\text { Strength } \\
a_{g} / a_{r}\end{array}$ & $\begin{array}{c}\text { Fitting } \\
\text { range }(\mathrm{K})\end{array}$ & $a_{r}$ & $b_{r}$ \\
\hline $1.250 \mathrm{~cm}^{3} / \mathrm{g}$ & $100-240$ & $0.662 \pm 0.013$ & $-85.2 \pm 2.3$ & 276 & 97.5 & 1.38 & $320-500$ & $0.911 \pm 0.015$ & $-154.0 \pm 6.0$ \\
\hline $1.275 \mathrm{~cm}^{3} / \mathrm{g}$ & $100-220$ & $0.656 \pm 0.011$ & $-148.7 \pm 1.8$ & 249 & 14.3 & 1.33 & $280-500$ & $0.870 \pm 0.007$ & $-202.4 \pm 2.7$ \\
\hline
\end{tabular}




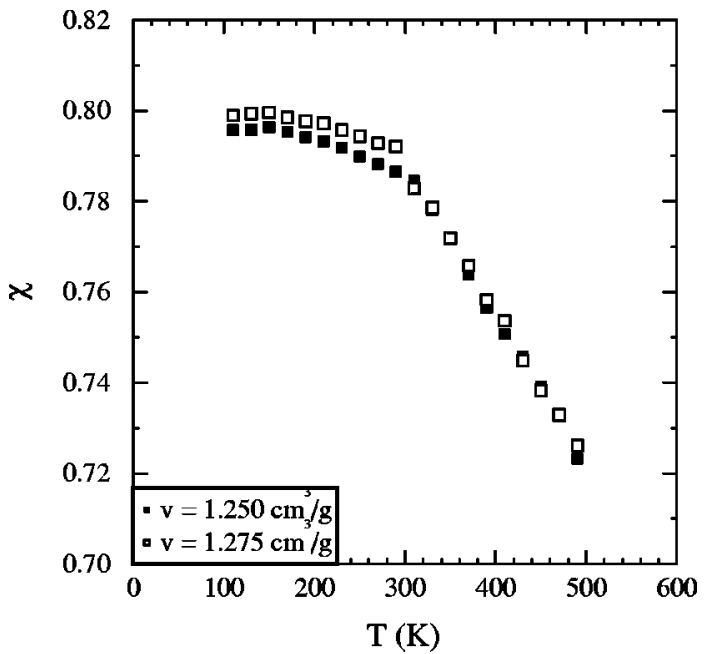

FIG. 6. The trans fraction $\chi$ vs temperature for $v=1.250$ and $1.275 \mathrm{~cm}^{3} / \mathrm{g}$ isochoric simulations.

LJ potential energy with temperature during isochoric cooling are shown in Fig. 8. The bending energy varies linearly with temperature and shows no discernible change in this trend at temperatures near $T_{g}$. This is consistent with the observations made in the isobaric study and is indicative of the fact that the bending potential is in or near equilibrium both above and below $T_{g}$. The torsional energy varies linearly with temperature in both the rubbery and glassy states, but with a pronounced change in slope near $T_{g}$. This reflects the slowing of torsional angle changes as represented by $\chi$ shown in Fig. 6 and is consistent with the $E_{\text {torsion }}$ vs $T$ trends in the isobaric study (Fig. 4). These data again illustrate the freezing of the torsional conformational state change during the glass transition. The variation of the nonbonded LJ potential energy with temperature exhibits a very similar form to that for the temperature dependence of the pressure shown in Fig. 5. It varies linearly with temperature both above and below $T_{g}$, but with a pronounced change in slope at $T_{g}$. The fact that LJ potential energy for smaller specific volume $v=1.250 \mathrm{~cm}^{3} / \mathrm{g}$ is lower than that of $v=1.275 \mathrm{~cm}^{3} / \mathrm{g}$ indicates that the LJ potential energy is dominated by the repulsive part of the potential.

\section{DISCUSSION}

The isobaric and isochoric glass formation simulations reproduce many of the salient features of the experimental observations. In general, physical phenomena can only be observed when the temporal and spatial scale of the processes are comparable-compatible with those of the observation technique. The strong correlation between the experimental observations and the present simulation results on isobaric and isochoric glassy transitions demonstrate that the central physics is occurring on scales no larger than those probed by the simulations: $\sim 5 \mathrm{~nm}$ and $\sim 1$ nanosecond. Furthermore, while the cooling rates in the simulation are extraordinarily fast $\left(2^{\circ} \mathrm{K} /\right.$ picosecond), the same type of relaxations that occur at normal cooling rate also occur here, although, perhaps, shifted to shorter length scales and higher temperatures.
Glass transition phenomena all have their origins in the basic atomic interactions. The isobaric-isochoric glass formation simulations showed that both the specific volume/ pressure (Figs. 1 and 5) and trans-state fraction $\chi$ (Figs. 3 and 6) undergo an abrupt change at nearly the same temperature. The specific volume-pressure is directly related to the nonbonded Lennard-Jones potential (as is easily seen by noting that the isostructural dilatation of the glass affect this part of the potential and not the bending or torsional components). The trans-state fraction, on the other hand, is directly controlled by the torsional potential. Given these relationships, we see that the glass transition is controlled primarily by the nonbonded and torsional parts of the atomic interactions. This is explicitly born out by the observations that both the nonbonded and torsional (Figs. 4 and 7) parts of the internal energy undergo abrupt changes at nearly the same temperature (i.e., $T_{g}$ ) as do the specific volume-pressure (Figs. 1 and 5) and trans-state fraction $\chi$ (Figs. 3 and 6). The fact that the specific volume-pressure and torsional degrees of freedom become more restricted at the same temperature suggests that these two types of degrees of freedom are coupled to each other. Although the bending potential change does not appear to be directly correlated with the glass transition, the stiff bending potential and the bond length constraint together define the connectivity of the polymer system and are responsible for the behavior that is directly tied to the macromolecular structure. In this sense, all of the components of the interatomic bonding conspire to determine the glass transition properties of polymeric systems.

Comparison of Figs. 4 and 7 show that the torsional potential energy exhibit similar variations with temperature in both the isobaric and isochoric cases. This indicates that the torsional degree of freedom undergoes similar changes upon cooling through the glass transition in both cases. On the other hand, the magnitude of the variations in the nonbonded LJ potential during isochoric cooling (Fig. 7) is only approximately half as large as in the isobaric case (Fig. 4). The contribution of the LJ potential to the strength of the glass transition, as measured by the ratio of the slopes of the LJ energy above and below the glass transition, is significantly greater upon isobaric cooling $(2.0,1.9$, and 1.8 for $P=0,100$, and $200 \mathrm{MPa}$, respectively) than upon isochoric cooling (1.5 for both specific volumes). This effect may be responsible for the weaker overall isochoric glass formation strength (defined as the pressure slope change) as compared with that under isobaric conditions (defined as the specific volume slope change).

We may understand the weaker glass transition under isochoric conditions as follows. The pressure, $P=\sum_{\alpha=1}^{N}\left(\mathbf{P}_{\alpha}^{2} / M+\mathbf{F}_{\alpha} \mathbf{R}_{\alpha}\right)(3 V)^{1}=N k_{B} T / V+\sum_{\alpha=1}^{N}\left(\mathbf{F}_{\alpha} \mathbf{R}_{\alpha}\right)$ $\times(3 V)^{-1}$, has contributions from both thermal motion (first term) and the atomic interactions (second term), where the sum on $\alpha$ is over all molecules. Under isobaric conditions, decreasing the temperature results in a decrease in volume. This can be seen using equation above, where decreasing the temperature at fixed pressure necessarily implies that the second term on the right hand side must increase. Since this term is dominated by the nonbonded LJ interactions, it in- 

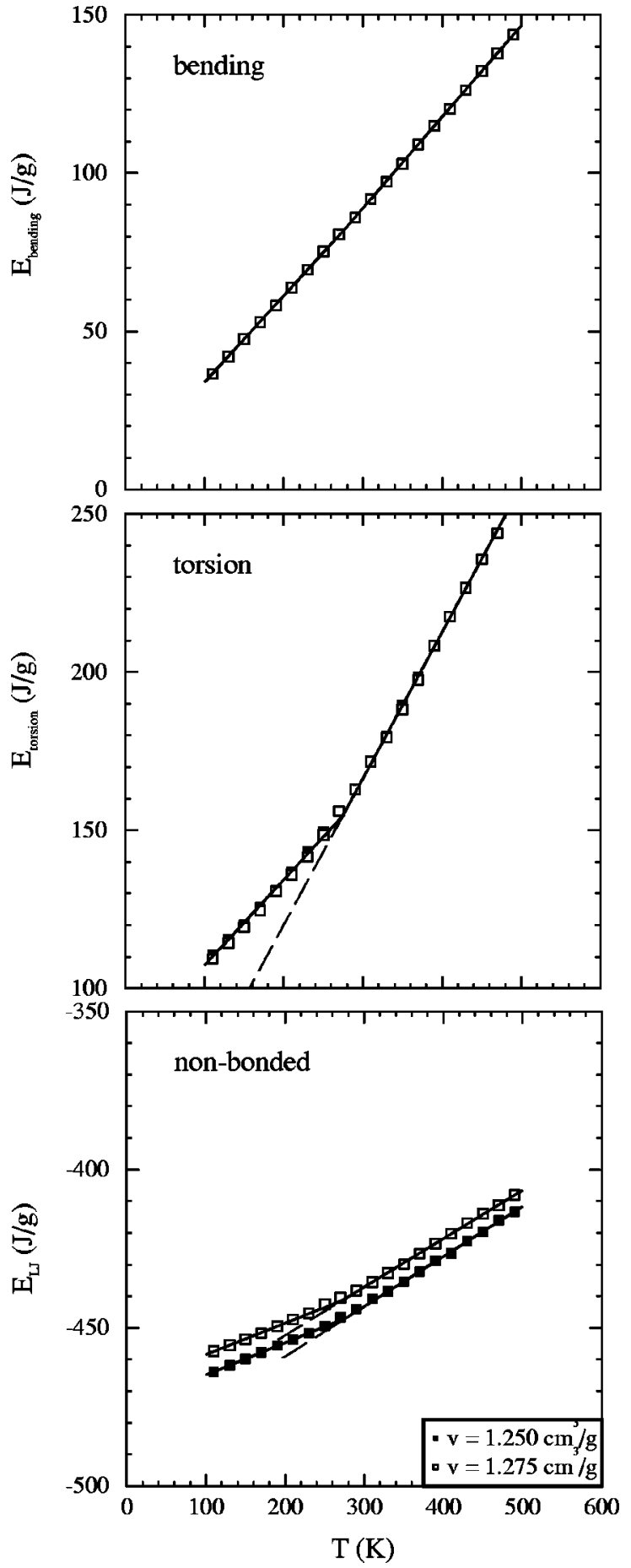

FIG. 7. The bending, torsional, and nonbonded LJ energies vs temperature. The symbols represent the simulation data and the solid lines represent least square linear fits to the data in the rubbery and glassy states. The dashed lines are extrapolations of the high temperature fits to below $T_{g}$. The same temperature ranges were used for the fitting procedure here as in Fig. 5 (see Table II).

creases when the specific volume decreases. This also explains why the nonbonded LJ energy is a much weaker function of temperature under isochoric, rather than isobaric, conditions (cf. Figs. 4 and 7). The reason that the nonbonded $\mathrm{LJ}$ interactions changes at all at fixed volume above $T_{g}$ is that decreased thermal fluctuations at low temperature mean that adjacent chains fluctuate into close proximity (high LJ

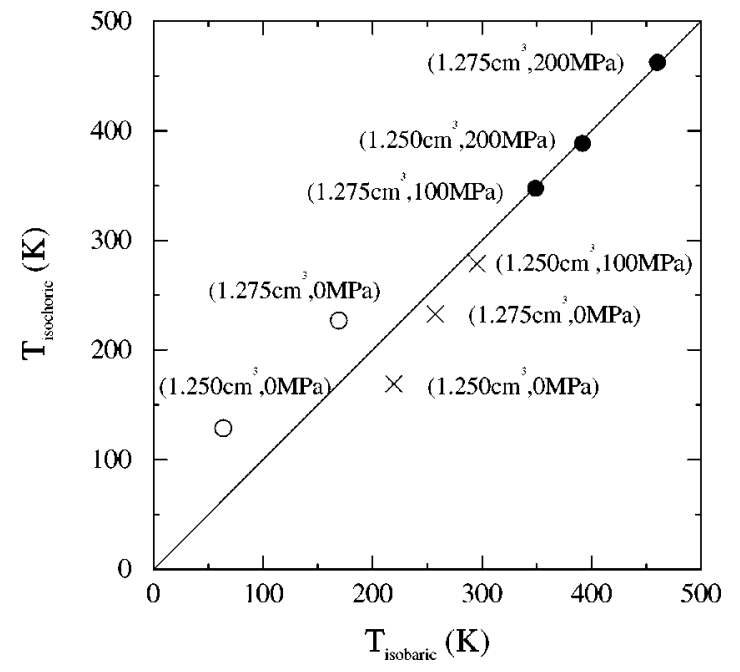

FIG. 8. The temperatures at which the indicated pressure and volume state were obtained via isobaric $T_{\text {isobaric }}$ and isochoric $T_{\text {isochoric }}$ cooling. The filled and open circles represent states above and below $T_{g}$, respectively. The X's represent data obtained by linearly extrapolating the data from above to below $T_{g}$.

energy) less frequently. Since the glass transition corresponds to a dramatic slowing of the torsional degrees of freedom (see Figs. 3 and 6), the motions that occur below $T_{g}$ are limited to small torsional angle fluctuations, with few torsional (trans vs gauche) state changes. The LJ energy reflects the fact that the molecular coordinates are no longer in equilibrium. Hence, the LJ energy versus temperature curve below $T_{g}$ is above the extrapolation of the high temperature, equilibrium LJ energy (see, Figs. 4 and 7). In the isobaric case, the deviation of the LJ energy vs temperature curve below $T_{g}$ from the extrapolation of the high temperature LJ energy is even more pronounced. This is because in addition to freezing the structure below $T_{g}$, the volume also falls out of equilibrium (see Fig. 1). In conclusion, the glass transition is stronger under isobaric conditions than under isochoric conditions because in the latter case only the structure falls out of equilibrium at $T_{g}$, while in the former both the structure and volume are nonequilibrium.

The trans-state fraction and potential energy data suggest that the glass transition is primarily associated with the freezing of the torsional degrees of the polymer chains. This picture we have described for the glass transition is consistent with those theories using configurations entropy as the basis for the transition, viz., the Adam-Gibbs and GibbsDiMangio theories. ${ }^{9}$ Our picture is also consistent with these theories in that the decreased volume is just part of the reasons that permit fewer chain configurations.

The thermodynamic properties of an equilibrium system are independent of the manner in which the system was prepared. In the present study, we prepared glasses through isobaric and isochoric cooling. In a few cases, these data overlap such that we have two sets of samples at the same pressure and volume. In Fig. 8, we plot the temperatures at which the isochoric and isobaric systems have the same $P$ and $v$. The solid circles represent both the isobaric and isochoric states at temperatures above $T_{g}$. These data fall upon a straight line of unit slope (i.e., $T_{\text {isochoric }}=T_{\text {isobaric }}$ ). This 
implies that above $T_{g}$, the rubbers produced by isobaric and isochoric cooling are indistinguishable and, hence, in thermodynamic equilibrium. The data point corresponding to $v=1.275 \mathrm{~cm}^{3} / \mathrm{g}$ and $P=100 \mathrm{MPa}$ corresponds to a temperature that is only $\sim 25^{\circ}$ above the isobaric $T_{g}$, suggesting that equilibrium was obtained down to very close to the isobaric $T_{g}$. The open circles in Fig. 8 represent isobaric and isochoric data for systems cooled to below their glass transition temperatures, in which case the data clearly deviate from the unit slope straight line. This implies that the glass structure is a nonequilibrium structure. The fact that the open circles sit above the solid line for the same $P$ and $v$, suggests that the glass must be cooled to a lower temperature under isobaric conditions to reach the same state as in the isochoric case. In other words, it is easier to form a dense glass along an isochoric path than along an isobaric one. This is consistent with the picture presented below which suggests that the amount of free volume in the system increases on cooling in the isochoric case and decreases on cooling in the isobaric case. The extra volume available in the isochoric case permits greater mobility at the same temperature and, hence, results in a more equilibrated glass. If it was possible to supercool the liquid below $T_{g}$, the thermodynamic state could be obtained by extrapolation of the high temperature data to below $T_{g}$. Like for the data obtained above $T_{g}$, we might expect that the isochoric and isobaric extrapolations below $T_{g}$ would produce the same "equilibrium" state. Figure 8 shows the results of such isobaric and isochoric linear extrapolations to the same $P$ and $v$ (indicated by the symbol " $X$ "). These data show that such linear extrapolations do not produce the same thermodynamic state (i.e., data fall off the unit slope straight line). One possible explanation of this apparent failure is that the linear extrapolation of the high temperature data to below $T_{g}$ is not valid for the isochoric, isobaric, or either trajectory. All of this data can be pushed onto the straight line of unit slope by admitting a small amount of curvature to the $v$ vs $T$ (positive $v^{2}$ coefficient) or $P$ vs $T$ (negative $P^{2}$ coefficient) extrapolations. While this is consistent with some existing isobaric data (e.g., Chap. 1 of Ref. 1) and our simulations, we have no physical rationale upon which to base this choice.

Many commonly observed polymer phenomena, including the glass transition, are rationalized on the basis of free volume (e.g. Refs. 1 and 2). Although a critical examination of the free volume theory is beyond the scope of this study, we can analyze the simulation results in terms of free volume theory, as per the Colucci et al. ${ }^{16}$ analysis of isobaric and isochoric experimental data. According to the free volume theory of Fox and Flory, ${ }^{5} T_{g}$ can be viewed as that temperature below which insufficient free volume exists for molecules to adjust. This gives rise to the concept of a critical volume. Below $T_{g}$, the quantity of free volume remains fixed, as does its spatial arrangement. The relatively small changes in volume observed below $T_{g}$ results from thermal vibrations. Above $T_{g}$, volume increases with temperature due to both thermal vibrations and the increasing free volume. The free volume $V_{f}$ at or below the glass transition under isobaric conditions can be expressed as $V_{f}=V_{g}-V_{0}$ $-(\partial V / \partial T)_{g} T_{g}$, where $V_{g}$ is the volume at $T_{g}, V_{0}$ is the oc-

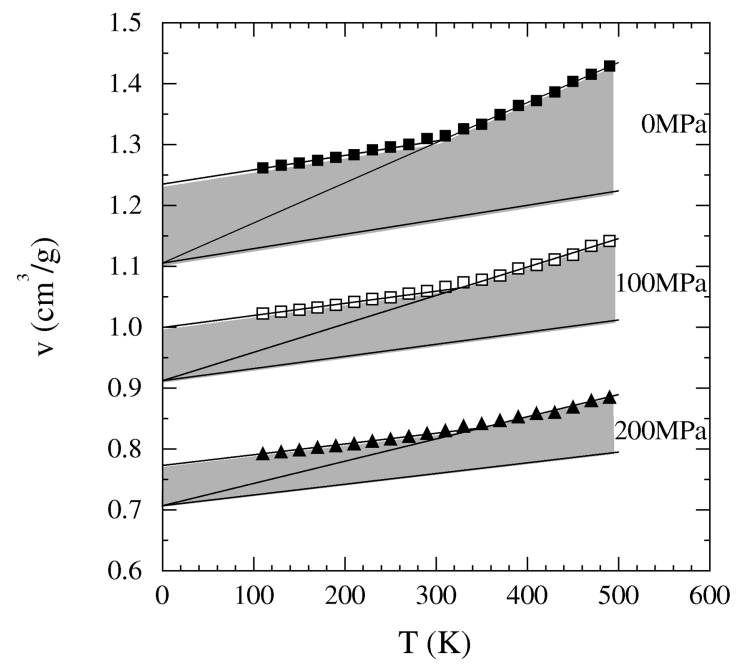

FIG. 9. The volume as a function of temperature for $P=0,100$, and 200 $\mathrm{MPa}$ (for clarity, the $P=100$ and $200 \mathrm{MPa}$ were shifted by -0.2 and -0.4 $\mathrm{cm}^{3} / \mathrm{g}$, respectively). The high and low temperature data were extrapolated to $T=0$ and a line with the slope of the low temperature data was constructed starting at the volume corresponding to the extrapolation of the high temperature data. The difference between the volume and this line (i.e., the shaded region) is a measure of the free volume, as described in the text.

cupied volume at temperature zero and the derivative is evaluated in the glassy state (p. 172 of Ref. 1). The free volume above $T_{g}$ can be expressed as: $V_{f}=V_{g}-V_{0}$ $-(\partial V / \partial T)_{g} T_{g}+\left[(\partial V / \partial T)_{r}-(\partial V / \partial T)_{g}\right]\left(T-T_{g}\right)$, where the subscript $r$ indicates that the derivative is evaluated in the rubbery state. Simha and Boyer ${ }^{6}$ suggested that $V_{0}$ is the hypothetical volume of the liquid state extrapolated to zero temperature from above $T_{g}$. Our isobaric simulation data show that for all pressures examined, a linear extrapolation of the volume from above $T_{g}$ to $T=0$ yields the same volume $v=1.11 \mathrm{~cm}^{3} / \mathrm{g}$ (Fig. 1), we set $v_{0}=1.11 \mathrm{~cm}^{3} / \mathrm{g}$. We determine the free volume under isobaric conditions following the procedure described above and illustrate the analysis at three pressures in Fig. 9. The height of the shaded area in this plot represents the free volume. The free volume at the glass transition is $0.129,0.087$, and $0.063 \mathrm{~cm}^{3} / \mathrm{g}$ at $P=0,100$, and $200 \mathrm{MPa}$, respectively. Clearly, the free volume at $T_{g}$ is not a constant and is, in fact, a strong function of pressure. Therefore, the assumption that the glass transition occurs at a critical free volume is not universally valid.

We now examine the variation of the free volume under isochoric conditions. The variation of the total volume has contributions from thermal fluctuations and the variation of the free volume with temperature. The total volume remains fixed in the isochoric case. Hence, the changes in the thermal fluctuation and free volume contributions to the total volume with changes in temperature must be equal and opposite. Since the thermal fluctuation component must decrease with decreasing temperature (it classically goes to zero as the temperature goes to absolute zero), we must conclude that the free volume increases under isochoric cooling. Hence, the free volume model would suggest that isochoric cooling of a rubber would never produce a glass. This seems to contradict both the present simulation and experimental results of Colucci, et al. ${ }^{16}$ 
The myriad successes of the free volume theory make us reluctant to dismiss wholesale this approach despite its apparent failure. Therefore, we must view the free volume model as part of a more general picture. Free volume provides one measure of the local environment. Clearly, there are other measures not represented by this single parameter. For example, local topology and polarization of the media constitute nonfree volume descriptions of the local environment. Local structure measures are important in as much as molecular motion and local structure are interdependent. In the free volume picture of the glass transition, free volume is required to provide room for local structural transitions (e.g., trans-gauche) to take place. The possibility of other structural descriptors which may affect molecular motions suggest that the presence of sufficient free volume may be a necessary, although not sufficient condition for molecular motion. For example, simply because sufficient free volume exists to allow trans-gauche transitions, the barrier to such transitions may be sufficiently large that they will not occur at a particular temperature. This opens the possibility that the isochoric cooling results, in which a glass transition occurs during an increase in free volume, may be associated with another mechanism for restricting molecular motions. A corollary is that if we provide a means by which to overcome one set of local restrictions (e.g., by raising the temperature), then either another class of local restriction will dominate or the system will equilibrate. Many viscoelastic and transport processes in polymer glasses are explained in terms of holes that are free to redistribute (note that in this sense free does not mean merely unoccupied but instead free to move). The present results suggest that the redistribution is a result of conformational transitions and the "free" volume should be viewed as a convenient description of the molecular mobility, not the cause for mobility, as is sometimes done.

\section{CONCLUSIONS}

We have performed molecular dynamics simulations of the glass transition through isobaric and isochoric cooling of a model polymeric material. Under isobaric conditions, the specific volume decreases linearly with decreasing temperature both above and below $T_{g}$, with an abrupt change of slope indicating the glass transition. Increasing pressure results in an increase in $T_{g}$ and a decrease in the specific volume at $T_{g}$. Glasses prepared at different pressures have specific volumes that differ by more than that expected on the basis of the compressibility of the material, indicating that these nonequilibrium structures are path dependent. The percentage of the torsional angles corresponding to the trans orientation $\chi$ in the rubbery state increases during cooling and is approximately pressure independent. Below $T_{g}, \chi$ is temperature insensitive, but pressure dependent. The bending energy decreases on cooling and shows no signature of the glass transition. The torsional energy of the rubbery material decreases upon cooling and is pressure independent and undergoes an abrupt, pressure dependent change at the glass transition temperature. The nonbonded Lennard-Jones potential energy decreases linearly with decreasing temperature both above and below $T_{g}$ with an abrupt change in slope at $T_{g}$. This energy contribution varies with pressure both above and below $T_{g}$. Under isochoric conditions, the pressure decreases linearly with decreasing temperature and exhibits an abrupt change in slope at $T_{g}$. Increasing specific volume leads to a decrease in $T_{g}$ and the pressure at $T_{g}$. The trans state fraction and the individual components of the potential energy exhibit similar variations with temperature as in the isobaric cooling case. However, the glass transition is considerably weaker under isochoric conditions than under isobaric conditions.

The trans-state fraction and potential energy data suggest that the glass transition is primarily associated with the freezing of the torsional degrees of the polymer chains. This freezing is strongly coupled to the degree of freedom associated with the nonbonded LJ potential. The greater strength of the glass transition under isobaric conditions as compared with isochoric cooling is associated with the fact that the nonbonded LJ potential is sensitive to the specific volume, which is constant during isochoric cooling. We find that the thermodynamic state is independent of the cooling path above $T_{g}$, while path dependent below $T_{g}$. We were able to extract the free volume from our simulation data, based on a linear extrapolation of the rubbery state data to $T=0$. These data clearly show that the free volume at the isobaric glass transition temperature is pressure dependent. Furthermore, a glass transition occurs under isochoric conditions, even though the free volume actually increases with decreasing temperature. Both of these results clearly contradict to the various modification of free volume glass transition theories. Therefore, we conclude that the free volume description of the glass transition is incomplete and other aspects of the local structure play perhaps more important roles in the glass transition phenomena.

\section{ACKNOWLEDGMENTS}

We gratefully acknowledge the financial support from the National Science Foundation (Grant No. DMR9422049) and computational resources from the Center of Parallel Computing of the University of Michigan. We also extent our thanks to Professor Andre Lee of Michigan State University for stimulating discussion and making a copy of their manuscript available to us before publication.

${ }^{1}$ R. N. Haward, The Physics of Glassy Polymers (Wiley, New York, 1973).

${ }^{2}$ R. N. Haward and R. J. Young, The Physics of Glassy Polymers, 2nd ed. (Chapman \& Hall, 1997).

${ }^{3}$ Kurt Binder, Monte Carlo and Molecular Dynamics Simulations in Polymer Science (Oxford University, New York, 1995).

${ }^{4}$ G. B. McKenna, in Comprehensive Polymer Science, Vol 2, Polymer Properties, edited by C. Booth and C. Price (Pergamon, Oxford, 1989).

${ }^{5}$ T. G. Fox and P. J. Flory, J. Am. Chem. Soc. 70, 2384 (1948); J. Appl. Phys. 21, 581 (1950).

${ }^{6}$ R. Simha and R. F. Boyer, J. Chem. Phys. 37, 1003 (1962).

${ }^{7}$ M. I. Williams, R. F. Landel, and J. D. Ferry, JACS 77, 3701 (1955).

${ }^{8}$ A. K. Doolittle, J. Appl. Phys. 22, 471 (1951).

${ }^{9}$ J. H. Gibbs and E. A. DiMarzio, J. Chem. Phys. 28, 373 (1958).

${ }^{10}$ D. Rigby and R.-J. Roe, J. Chem. Phys. 87, 7285 (1987).

${ }^{11}$ R.-J. Roe, D. Rigby, F. Hidemine Furuya, and H. Takeuchi, Comput. Polym. Sci. 2, 32 (1992), and papers cited therein.

${ }^{12}$ D. Brown and J. H. R. Clarke, Macromolecules 24, 2075 (1991).

${ }^{13}$ P. V. K. Pant, J. Han, G. D. Smith, and R. H. Boyd, J. Chem. Phys. 99, 597 (1993).

${ }^{14}$ R. H. Boyd, R. H. Gee, J. Han, and Y. Jin, J. Chem. Phys. 101, 788 (1994). 
${ }^{15}$ J. Han, R. H. Gee, and R. H. Boyd, Macromolecules 27, 7781 (1994).

${ }^{16}$ D. M. Colucci, G. B. McKenna, A. Lee, D. B. Curliss, K. Bowman, and J.

D. Russell, J. Polym. Sci., Polym. Phys. Ed. 35, 1561 (1997), and papers cited therein.

${ }^{17}$ H. C. Andersen, J. Chem. Phys. 72, 2384 (1980).

${ }^{18}$ S. Nosé, Mol. Phys. 52, 255 (1984).

${ }^{19}$ J. P. Ryckaert, G. Ciccotti, and H. J. C. Berendsen, J. Comput. Phys. 23, 327 (1977).
${ }^{20}$ J. P. Ryckaert and G. Ciccotti, J. Chem. Phys. 78, 7368 (1983).

${ }^{21}$ M. Ferrario and J. P. Ryckaert, Mol. Phys. 54, 587 (1985).

${ }^{22}$ L. Yang, D. J. Srolovitz, and A. F. Yee, J. Chem. Phys. 107, 4396 (1997).

${ }^{23}$ G. Ciccotti and J. P. Ryckaert, Comput. Phys. Rep. 4, 345 (1986), Appendix A.

${ }^{24}$ J. I. McKechnie, D. Brown, and J. H. R. Clarke, Macromolecules 25, 1562 (1992).

${ }^{25}$ K. V. Von Hellwege, W. Knappe, and P. Lehmann, Kolloid Z. Z. Polym. 183, 110 (1962). 